\title{
QAPV: A POLYNOMIAL INVARIANT FOR GRAPH ISOMORPHISM TESTING
}

\author{
Valdir Agustinho de Melo ${ }^{1}$, Paulo Oswaldo Boaventura-Netto ${ }^{2 *}$ \\ and Laura Bahiense ${ }^{3}$
}

Received September 21, 2011 / Accepted October 8, 2012

\begin{abstract}
To each instance of the Quadratic Assignment Problem (QAP) a relaxed instance can be associated. Both variances of their solution values can be calculated in polynomial time. The graph isomorphism problem (GIP) can be modeled as a QAP, associating its pair of data matrices with a pair of graphs of the same order and size. We look for invariant edge weight functions for the graphs composing the instances in order to try to find quantitative differences between variances that could be associated with the absence of isomorphism. This technique is sensitive enough to show the effect of a single edge exchange between two regular graphs of up to 3,000 vertices and 300,000 edges with degrees up to 200. Planar graph pairs from a dense family up to 300,000 vertices were also discriminated. We conjecture the existence of functions able to discriminate non-isomorphic pairs for every instance of the problem.
\end{abstract}

Keywords: graph isomorphism, quadratic assignment problem, variance.

\section{INTRODUCTION}

For each discussion presented in this work, the fundamental graph-theoretical concepts can be found in Harary [19] and Gross \& Yellen [18]. A preliminary paper on the same subject, with abridged tests, is Melo et al. [26].

The graph isomorphism problem has been studied by many researchers, owing to its theoretical interest and from its possible applications, such as pattern recognition, De Piero \& Krout [10]. Let $G_{1}=\left(V_{1}, E_{1}\right)$ and $G_{2}=\left(V_{2}, E_{2}\right)$ be two simple graphs with independent labelings of their vertex sets. Then $G_{1}$ and $G_{2}$ are isomorphic if and only if there is a bijection $\varphi: V_{1} \leftrightarrow V_{2}$ that preserves their adjacency relations. The general problem arises when $G_{1}$ is a graph and $G_{2}$ is a subgraph of another graph with at least the same order $n$ and size $m$ of $G_{1}$. A particular case, which the present work addresses, is the restricted problem, which we will abbreviate as GIP.

\footnotetext{
*Corresponding author

${ }^{1}$ Program of Production Engineering, COPPE/UFRJ, Brazil. Post-doctoral researcher. E-mail: vmelo@pep.ufrj.br

${ }^{2}$ Universidade Federal do Rio de Janeiro/COPPE, Brazil. Full Professor, retired; Program of Production Engineering, COPPE/UFRJ. E-mail: boaventu@pep.ufrj.br

${ }^{3}$ Program of Production Engineering, COPPE/UFRJ, Brazil. Associate Professor. E-mail: laura.bahiense@gmail.com
} 
It is the problem of matching two graphs of the same order and size. It is is NP, but to date no one has been able to say if it is polynomial or NP-complete for every graph pair, Garey \& Johnson [15], Arvind \& Thoran [2].

The study of graph isomorphism has been done with the aid of two general classes of resources:

- matching algorithms, which look for building a graph in a way that matches an isomorphism bijection, if it exists: McKay [27] proposed a specific one; Cross et al. [8] and Porumbel [29] use metaheuristics. DePiero \& Krout [10] uses path counts to approximate subgraph isomorphism. Foggia et al. [14] is a comparison among five commonly used algorithms. Gori et al. [16] use random walks. Jain \& Wyzotski [22] uses neural nets; Ding \& Huang [11] reorganize the graph in searching for a perimeter and a canonical adjacency matrix. Dharwadter \& Tevet [13] presents a polynomial algorithm for the GIP, but Santos [31] found a counterexample for it. Presa [PR09] is a thesis on GIP algorithms. Czerwinski [9] is a theoretical paper which proposes a polynomial algorithm. Voss \& Subhlock [34] is a performance comparison based on some graph classes, from 8 to 16,000 nodes. Douglas [12] discusses the possibility of applying the Weisfeiler-Lehman algorithm to the GIP, raising some open questions.

- efficient invariants. A graph parameter is (an) invariant if it has the same value for every isomorph of a given graph. The most readily available invariants are naturally the order $n$ and the size $m$, but one would like to have an invariant where preserving value would be a necessary and sufficient condition for isomorphism - which is not, precisely, the case of order and size. An important invariant to consider is the ordered degree set (ODS) associated with a graph but, once again, two graphs with the same ODS can be non-isomorphic. On the other hand, two graphs with different ODS are non-isomorphic - and ODS is easy to calculate through a polynomial ordering algorithm. The graph spectrum (its set of adjacency matrix eigenvalues) is also an invariant but, again, there are non-isomorphic cospectral graph pairs, Cvetkovic et al. [7]. Until now, necessary and sufficient invariance is an open research field, no invariant having been found which fulfills it. Most wellknown invariants (such as the chromatic number and the independence number) are not polynomial, thus they are of no interest here.

This work proposes an invariant (the $\boldsymbol{Q A P V}$ invariant) to be calculated using the Quadratic Assignment Problem (QAP) structure (Loiola et al. [24]). Our aim is to translate into a non-zero deviation value any structural difference between two graphs of the same order and size. If we obtain different values of this invariant for the two graphs, they will certainly be non-isomorphic: we say that we have been able to discriminate between the two graphs. We present the results by giving a gap between two values associated with the graphs.

The database for a QAP instance is a pair of (symmetric) matrices $\boldsymbol{F}$ and $\boldsymbol{D}$, which are frequently associated with work flows between pairs of machines and distances between machine locations. For given $\boldsymbol{F}$ and $\boldsymbol{D}$, the pair $(\boldsymbol{F}, \boldsymbol{D})$ is a $Q A P$ instance, QAP $(\boldsymbol{F}, \boldsymbol{D})$. Among a number of possible 
meanings, a QAP instance can be constructed with the adjacency matrices of two simple graphs of equal order and size, which can be used to investigate isomorphism.

Despite the QAP's NP-hardness, the calculation of the variance of a QAP instance's solution set is polynomial, Boaventura-Netto \& Abreu [4], Abreu et al. [1]. Given a graph pair (G,H), we can define two more instances, $(\mathbf{G}, \mathbf{G})$ and $(\mathbf{H}, \mathbf{H})$, and calculate these three variances. If $\mathbf{G}$ and $\mathbf{H}$ are isomorphic, they have to be equal, but this is not sufficient for the graphs to be isomorphic: a counterexample is the pair (Petersen graph, pentagonal prism) in Figure 1.1.
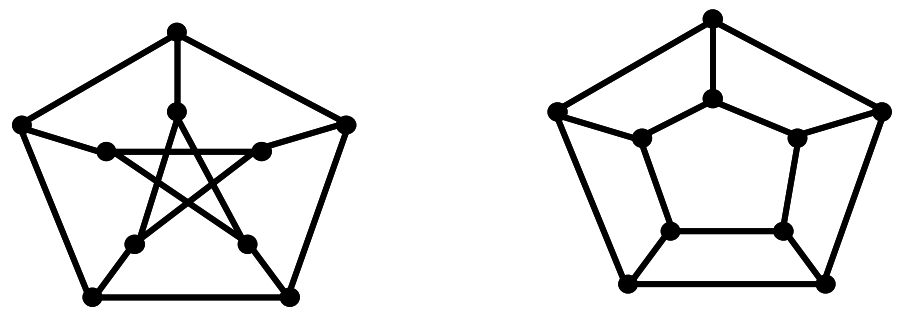

Figure 1.1 - Petersen graph and pentagonal prism.

To deal with this difficulty, we use invariant edge weight functions: functions on the edge set whose values depend only on the graph structure and so are independent of the vertex numbering, thus reinforcing eventual differences between the graph structures. In this work, we present some such functions, discuss their complexity and apply them to sets of examples of regular and planar graphs and also to pairs obtained from public databases. The use of regular graphs has to do with the difficulty to be foreseen in discriminating between a given graph and another with closely similar structure: one could expect to find general non-regular graphs an easier problem to work with. Among the planar graphs, we chose the triangulated-grid family (6-point stencils, Voss \& Subhlok [34], given by their order and rectangular $(p, q)$ dimensions). Their square diagonals are built in the northeast-southwest direction, as in [34]. These graphs are near the maximum density for planar graphs; they have $n=p q$ vertices and $m=3 n-2(p+q)+1$ edges. Thus, a $(600,500)$ graph of this type has almost 900,000 edges.

\section{THEORETICAL BASIS}

\subsection{Quadratic Assignment Problem (QAP)}

The QAP can be seen as the problem of how to assign $n$ activities to $n$ positions in such a way as to optimize the transportation cost between activity pairs over the distances associated with position pairs,

$$
z^{*}=\operatorname{opt}_{\varphi \in \Pi_{n}} \sum_{1 \leq i<j \leq n} f_{i j} d_{\varphi(i) \varphi(j)}
$$

Minimization is the most frequent application, but when dealing with graph isomorphism we will be interested in maximization. Matrix $\boldsymbol{F}=\left[f_{i j}\right]$ is the flow matrix (quantities transported between $i$ and $j$ ) and matrix $\boldsymbol{D}=\left[d_{k l}\right]$ is the distance matrix, where $d_{k l}$ is the distance between positions $k$ and $l$ ). In this work we consider $\mathbf{F}$ and $\mathbf{D}$ as symmetric matrices. 
The parcels of (2.1) are related to the set $\Pi_{n}$ of all $n$-element permutations, with every QAP solution being associated with a permutation $\varphi \in \Pi_{n}$, in this case, based on positions with respect to activities. Thus, QAP structure is evidently attractive when examining the restricted GIP: if $\mathrm{G}_{1}$ and $\mathrm{G}_{2}$ are isomorphic with adjacency matrices $\boldsymbol{A}_{1}$ and $\boldsymbol{A}_{2}$, we will find in the solution set of QAP $\left(\boldsymbol{A}_{1}, \boldsymbol{A}_{2}\right)$ a permutation corresponding to their isomorphism relation. We give further attention to this point in Item 2.3.

Since graph isomorphism preserves the adjacency relations, if we look for a maximum value $z^{*}$ of a solution for such an instance we will find either $z^{*}=m$, if the graphs are isomorphic, or $z^{*}<m$, if they are not. The first result is a necessary and sufficient condition for isomorphism, but we immediately run into a complexity problem: the QAP is NP-hard and experience shows its exact resolution is presently limited, with some exceptions, to instances with order below 40. Specially designed heuristics, as well as metaheuristics, have been applied to it. With these resources, graph pairs of higher order have been successfully tested for isomorphism by treating them as QAP instances, Lee et al. [25], the results being subjected to the error margin of the heuristics.

\subsection{Statistic moments of QAP instances}

Average solution value and variance of solution values have been used to compare the computational difficulty of QAP instances with respect to heuristic algorithms. We will be concerned with the results of Graves \& Whinston, [17] and Abreu et al. [1]. Let us discuss briefly the calculation presented there.

We can store the data of a symmetric QAP instance with the aid of two vectors, F and $\mathbf{D}$, with order $N=n(n-1) / 2$, respectively associated with the matrices $\boldsymbol{F}$ and $\boldsymbol{D}$, where a vector position $k$ can be associated with a matrix position $(i, j)$ by the expression

$$
k=(i-1) n-i(i+1) / 2+j .
$$

We can then define a $N$-order matrix, $\boldsymbol{Q}=\mathbf{F D}^{T}$, which contains every parcel composing every QAP solution $\varphi$ (n-order permutation). Since (2.1) shows us both $i$ and $j(i<j)$ with values between 1 and $n$, every QAP solution has $N$ parcels, which correspond to a set of independent positions in $\mathbf{Q}$. All $n$ ! solutions of a given instance can be found there among the whole $N$ ! sets of this type. A problem based on $\mathbf{Q}$ is therefore a relaxation of the original QAP, where not every set of independent positions corresponds to a feasible QAP solution. We will denote such relaxation as $Q A P(\mathbf{Q})$. We have also use for ordered vectors $\mathbf{F}^{-}$and $\mathbf{D}^{+}$obtained from $\mathbf{F}$ and D through non-decreasing and non-increasing orderings respectively, from which we define a matrix $\mathbf{Q}^{\prime}=\left(\mathbf{F}^{-}\right)\left(\mathbf{D}^{+}\right)^{T}$.

Let $\mathrm{S}$ be the sum of all $\mathbf{Q}$ elements. Then the average cost of an instance $Q A P(\mathbf{F}, \mathbf{D}),[1],[3]$, [4], [17], is

$$
\mu=S / N \text {. }
$$


The variance of the relaxed instance, $Q A P(\mathbf{Q})$, is, [1], [17],

$$
\sigma_{Q}^{2}=\frac{1}{N}\left(\sum_{i=1}^{N} f_{1}^{2}\right)\left(\sum_{i=1}^{N} d_{j}^{2}\right)+\frac{1}{N(N-1)}\left[\sigma^{2}\left(f_{i}\right)\right]\left[\sigma^{2}\left(d_{j}\right)\right]-\mu^{2}(i, j=1, \ldots, n)
$$

The variance of the (original) instance $Q A P(\mathbf{F}, \mathbf{D})$ is, [1], [4],

$$
\sigma_{F, D}^{2}=\left(S_{0}+S_{1}+S_{2}\right) / n !-\mu^{2},
$$

where

$$
\begin{aligned}
& S_{0}=4(n-4) ! \sum_{\cap_{0}} f_{i j} f_{r s} \sum_{\cap_{0}} d_{i j} d_{r s} \\
& S_{1}=(n-3) ! \sum_{\cap_{1}} f_{i j} f_{r s} \sum_{\cap_{1}} d_{i j} d_{r s} \\
& S_{2}=2(n-2) ! \sum_{1 \leq i<j \leq n} f_{i j}^{2} \sum_{1 \leq i<j \leq n} d_{i j}^{2}
\end{aligned}
$$

and

$$
\left|\cap_{0}\right|=C_{n-2,2},\left|\cap_{1}\right|=2(n-2)
$$

where $\cap_{k}(k \in\{0,1\})$ is the set of pairs $((i, j),(r, s))$ such that $|\{i, j\} \cap\{r, s\}|=k$.

The computational complexity of the first variance is thus $O\left(n^{2}\right)$, and that of the second is $O\left(n^{4}\right)$, in both cases, considering $\boldsymbol{F}$ and $\boldsymbol{D}$ as full matrices. By using adjacency lists, they become $O(m)$ and $O\left(\mathrm{~m}^{2}\right)$ respectively. The reuse of several terms gives way to a significant time economy, as shown by the pseudocode below, which includes the three-instance calculation referred to in Item 1 (Figure 2.1):

\subsection{Instance classes related to isomorphism}

We can define a $Q A P\left(\mathbf{F}^{-}, \mathbf{D}^{+}\right)$with coefficient matrix $\mathbf{Q}^{\prime}$ and then define a related instance class where the ordering of every pair $(\mathbf{F}, \mathbf{D})$ will result in the same $\mathbf{Q}^{\prime}$, [1],

$$
\operatorname{Relclass}\left(\mathbf{F}^{-}, \mathbf{D}^{+}\right)=\left\{Q A P(\mathbf{F}, \mathbf{D}) /\left(\mathbf{F}^{-}\right)\left(\mathbf{D}^{+}\right)^{T}=\mathbf{Q}^{\prime}\right\}
$$

By going into graph language, a QAP instance can be represented by two complete graphs $\mathbf{K}_{F}$ and $\mathbf{K}_{D}$, respectively edge-valued by the weight functions $w\left(\mathbf{K}_{F}\right)=\mathbf{F}$ and $w\left(\mathbf{K}_{D}\right)=D$.

We will then say that two edge-valued complete graphs $\mathbf{K}_{n}\left(V_{1}, E_{1}, F\right)$ and $\mathbf{K}_{n}\left(V_{2}, E_{2}, \mathbf{D}\right)$ are w-isomorphic if there is a permutation $\alpha \in \Pi_{n}$ such that $(i, j) \in \mathbf{E}_{1} \Leftrightarrow(\alpha(i), \alpha(j)) \in \mathbf{E}_{2}$ and $\mathbf{F}(i, j)=\mathbf{D}(\alpha(i), \alpha(j))$. We will then denote this isomorphism as $w\left(\mathbf{K}_{\mathbf{F}}\right) \approx w\left(\mathbf{K}_{\mathbf{D}}\right)$. $Q A P\left(\mathbf{F}_{1}, \mathbf{D}_{1}\right)$ and $Q A P\left(\mathbf{F}_{2}, \mathbf{D}_{2}\right)$ will thus be isomorphic $\left(Q A P\left(\mathbf{F}_{1}, \mathbf{D}_{1}\right) \approx Q A P\left(\mathbf{F}_{2}, \mathbf{D}_{2}\right)\right)$ if and only if $w\left(\mathbf{K}_{F 1}\right) \approx w\left(\mathbf{K}_{F 2}\right)$ and $w\left(\mathbf{K}_{D 1}\right) \approx w\left(\mathbf{K}_{D 2}\right)$. 


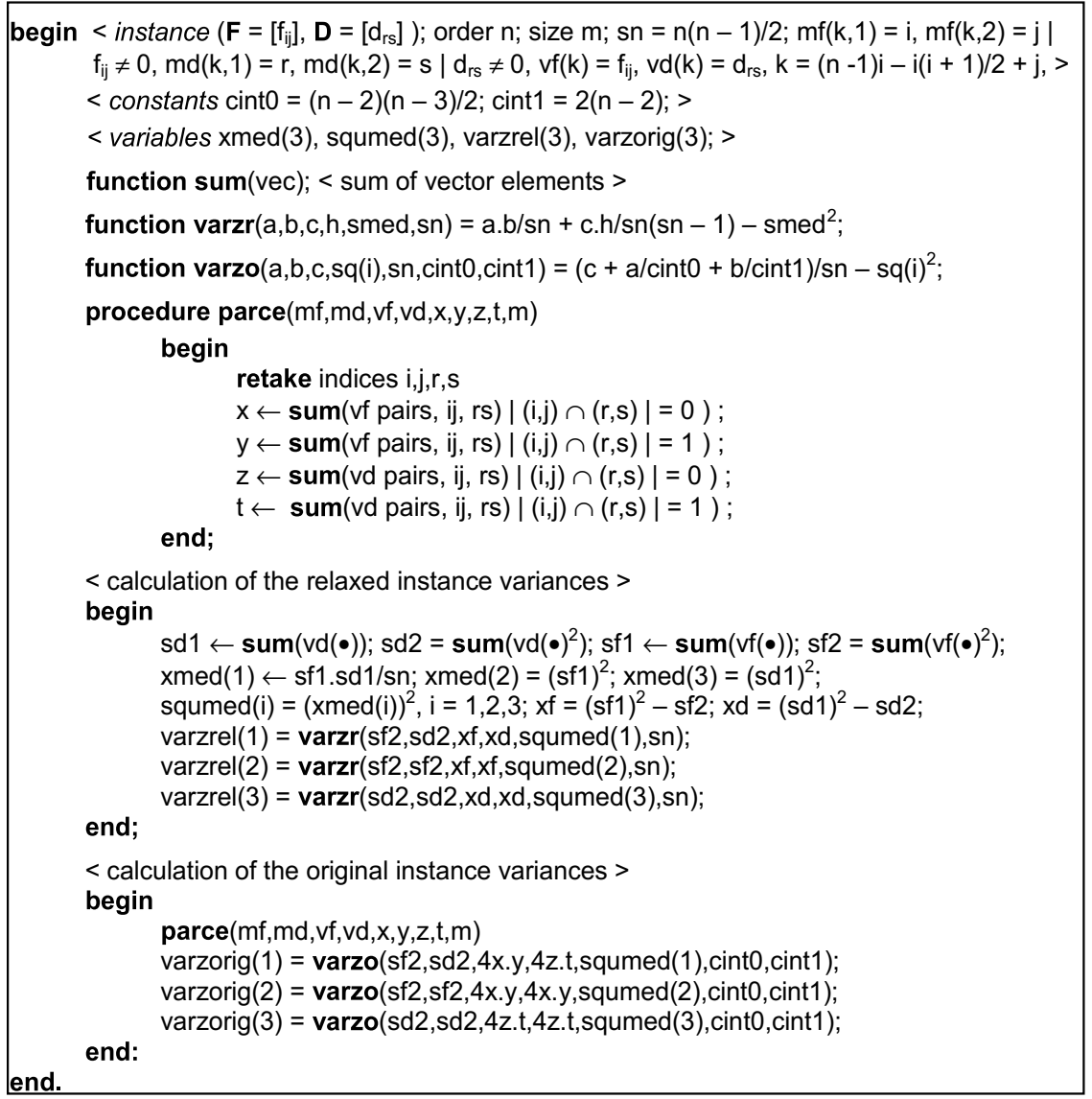

Figure 2.1 - Pseudocode for calculating the QAPV invariant.

On the other hand, we say that $\mathbf{G}_{1}=\left(\mathbf{V}_{1}, \mathbf{E}_{1}\right)$ and $\mathbf{G}_{2}=\left(\mathbf{V}_{2}, \mathbf{E}_{2}\right)$ are isomorphic if and only if there exists a function $f$ which preserves the adjacency relations over a permutation $\alpha$ of one of their vertex sets with respect to the other, that is, for every $(i, j) \in \mathbf{E}_{1}$ we have $f(\alpha(i), \alpha(j)) \in$ $\mathbf{E}_{2}$.

This isomorphism appears then as a particular case of the w-isomorphism defined in the context of QAP, where the weight function is given by the adjacency matrix for a graph $\mathbf{G}=(\mathbf{V}, \mathbf{E})$, $\mathbf{A}=\left[a_{i j}\right], a_{i j}=1$ if $(i, j) \in \mathbf{E}$ and $a_{i j}=0$ if $(i, j) \notin \mathbf{E}$.

The theorem and corollary below constitute the basis for the application of QAP variance on GIP.

Theorem 2.1 [1]: Two isomorphic instances $Q A P\left(\boldsymbol{F}_{1}, \boldsymbol{D}_{1}\right)$ and $Q A P\left(\boldsymbol{F}_{2}, \boldsymbol{D}_{2}\right)$ have the same set of feasible solutions.

This isomorphism implies that there is isomorphism between the corresponding relaxed instances, which may differ in $\mathbf{Q}$, but have the same $\mathbf{Q}^{\prime}$. However, not every pair of relaxed instances satisfying this condition is isomorphic. 
Corollary 2.2. Two isomorphic instances have the same variance.

Proof. Immediate from Theorem 2.1, since they share the same set of feasible solutions.

The variance of QAP solutions is then an invariant with respect to instance isomorphism. In Section 1, we had an initial look at the practical use of this property, but it requires a more detailed discussion.

Trying to avoid variance equality when using the comparison sketched in Section 1 with nonisomorphic graphs (such as those in Figure 1.1), we propose to give weights to the edges, thus obtaining matrices which could be tested for $w$-isomorphism. For this to have a meaning, these values have to be given by an invariant function - that is, a function whose values correspond to a property of the graph structure - which is given by its adjacency relations, preserved by isomorphism and not related to vertex labeling.

\section{METHODOLOGY}

\subsection{A comparison standard and the use of weight functions}

Let us call $Q A P\left(\mathbf{G}_{1}, \mathbf{G}_{2}\right)$ the instance built with the adjacency matrices of two graphs. We then associate two other instances with it, $Q A P\left(\mathbf{G}_{1}, \mathbf{G}_{1}\right)$ and $Q A P\left(\mathbf{G}_{2}, \mathbf{G}_{2}\right)$ with two copies of the same matrix for each graph. We will call these last two instances associated instances, and their variances, associated variances. Both for the original and the relaxed instances, Corollary 2.2 is only a necessary condition: to have the same variance is not sufficient for two instances to be isomorphic. A consequence of this is that a number of graph pairs with approximately similar structures show equal variances for the three instances presented above.

We try to overcome this difficulty by applying edge invariant weight functions, going into $w$ isomorphism to look for discrimination between the graphs of such pairs. In this work, we use the following functions, whose complexity is presented considering the use of adjacency lists. We will represent them as $f(\mathbf{G})$, where it is understood that the function concerns the edge set $\mathbf{E}$, for $\mathbf{G}=(\mathbf{V}, \mathbf{E})$.

- Function w3(G): number of 3-edge closed walks using the concerned edge: this function can be calculated in $\mathrm{O}(n d)$.

- Function $w 4(G)$ : number of 4-edge closed walks using the concerned edge: it is more costly since it deals with a larger neighborhood. The worst complexity case is $\mathrm{O}\left(n^{2} d\right)$.

These two functions can be calculated with the aid of the following theorem, the second one being obtained from the first one by induction:

Theorem 3.1 (Festinger, apud Boaventura-Netto [5]). Let $G=(V, E)$ be a graph, $\mathbf{A}=\left[a_{i j}\right]$ its adjacency matrix and $\mathbf{A}^{k}=\left[a_{i j}^{(k)}\right]$ the $k^{\text {th }}$ potency of $\mathbf{A}$. Then $a_{i j}^{(k)}$ is equal to the number of $k$-walks between $i$ and $j \in V$. 
Since we are interested in valuating pairs $i, j$ which define edges, the exponent to be used will be the length of the walks minus one $(k=2$ or 3 respectively to calculate $w 3(G)$ and $w 4(G))$. Other similar functions can be used, according to the girth of the graphs examined, but the complexity grows rapidly. We can also define new functions by summing the obtained values into the adjacency matrix, which can be beneficial with sparse graphs, thus making graph extensions in the sense of Porumbel [29].

- Function $\boldsymbol{d c}(\boldsymbol{G})$ : $\boldsymbol{d c}$ stands for distance to centers: it can be calculated with all-distances techniques such as Floyd or $n$-times applied Dijkstra algorithms. We use the distance matrix to find the graph center set $\mathbf{C}$ and then, for every $v \in \mathbf{V}$, we calculate $d c(v)=$ $\sum_{\bar{c} \in C} d(v, c)$. The function value for the edge $(i, j)$ is given by the sum of the values obtained for $i$ and $j$. The initial "infinite" values of non-existing edges will be greater than $n$. The complexity depends on the implementation, going from $\mathrm{O}\left(n^{2} \log n\right)$ to $\mathrm{O}\left(n^{3}\right)$.

- Function es $(\boldsymbol{G})$ : es stands for edge sum: for each vertex, it is the sum of its adjacent edge values. The edge function value is then the value sum of its adjacent vertices. es $(G)$ should not be directly applied to regular graphs (it would give equal values for all edges) but it can be applied to a regular graph already valued with the aid of another function, for example, $\boldsymbol{e s}(\boldsymbol{w} 3(\boldsymbol{G}))$. The complexity is $\mathrm{O}(m)$.

Other functions can be devised as discriminating tools, provided they are invariant and polynomial, as in Bessa et al. [6].

\subsection{Graph families used in the tests}

We used a regular graph generator where the vertex pair selection for edge introduction was randomly done with the aid of two random number generators, a conventional product-overflow generator and, for larger graphs, a $(5,17)$ lagged-Fibonacci generator, Marsaglia [28], Knuth [23]. The program contains an edge reallocation routine designed to correct regularity failures which are intrinsic to direct generation. Ten graphs of each order and degree were created this way.

We also generated what we call almost-isomorphic graphs by making random 2-exchanges in each original graph in order to create structural differences. We define a 2-exchange as an edge exchange in $\mathbf{G}=(\mathbf{V}, \mathbf{E})$ where we take off two edges $(\mathrm{a}, \mathrm{b}),(\mathrm{c}, \mathrm{d}) \in \mathrm{E}$ and replace them with either $(\mathrm{a}, \mathrm{c}),(\mathrm{b}, \mathrm{d}) \notin \mathrm{E}$, or $(\mathrm{a}, \mathrm{d}),(\mathrm{b}, \mathrm{c}) \notin \mathrm{E}$, thus preserving ODS. For each chosen $(n, d)$ pair, we constructed ten sets composed of an original graph and two almost isomorphs of it, comprising one graph with one 2-exchange and another with four 2-exchanges. We applied the weight functions to all these graphs. The data set has regular graphs with orders 50, 100, 200, 500, 1000, 1500, 2000, 2500 and 3000, with $d \leq n / 2$ and $d \in\{3,5,10,25,50,100,200\}$. Higher degree values were not used to generate the bigger graphs, in order to limit to acceptable values the processing times required by the weight functions.

The tests with planar graphs used five triangulated grids (TGG) for each order. In order to generate almost-isomorphs, we took each TGG and randomly selected four vertices from which 
we eliminated the northeast upper diagonal. The corresponding squares formed what we called windows in the graph, creating localized structure differences among them. To the graphs thus obtained we applied the function $e^{2}(\mathrm{G})$ and we took them two by two to build ten QAP instances. Order values and rectangle dimensions were 4,000 (50,80), 5,000 (50,100), 7,000 (70,100), 10,000 (100,100), 15,000 (150,100), 20,000 (200,100), 30,000 (200,150), 40,000 (200,200), $50,000(250,200), 60,000(300,200), 80,000(400,200), 100,000(400,250), 150,000(500,300)$, $200,000(500,400), 250,000(500,500)$ and 300,000 (600,500). All used databases can be found in $[5]$.

\subsection{Discrimination measure}

The discrimination instrument was the total percentage gap between the variance relation of the associated instances, $\sigma^{2}\left[Q A P\left(f\left(G_{1}\right), f\left(G_{1}\right)\right)\right]$ and $\sigma^{2}\left[Q A P\left(f\left(G_{2}\right), f\left(G_{2}\right)\right)\right]$, and that of the instance built with the graph pair, $\sigma^{2}\left(Q A P\left(f\left(G_{1}\right), f\left(G_{2}\right)\right)\right.$, calculated up to $10^{-12}$,

$$
\begin{aligned}
R \% G_{1} & =100\left\{\sigma^{2}\left[Q A P\left(f\left(G_{1}\right), f\left(G_{1}\right)\right)\right] / \sigma^{2}\left[Q A P\left(f\left(G_{1}\right), f\left(G_{2}\right)\right)\right]\right\} \\
R \% G_{2} & =100\left\{\sigma^{2}\left[Q A P\left(f\left(G_{2}\right), f\left(G_{2}\right)\right)\right] / \sigma^{2}\left[Q A P\left(f\left(G_{1}\right), f\left(G_{2}\right)\right)\right]\right\} \\
\operatorname{Gap}\left(G_{1}, G_{2}\right) & =\left|R \% G_{1}\right|+\left|R \% G_{2}\right| .
\end{aligned}
$$

From the previous discussion, a positive gap indicates absence of isomorphism while a null gap fails to discriminate between the pair of graphs. The results involve both the original and the relaxed instances. We know that $\operatorname{Relclass}\left(\mathbf{F}^{-}, \mathbf{D}^{+}\right)$includes non-isomorphic graphs, then it can happen that the relaxed instances calculation do not show discrimination. However, if we obtain this discrimination, it would be sufficient to guarantee the absence of isomorphism with a much lower computational cost. Most of our examples show this behavior, which can be seen in Tables 4.1 (a) and (b), where we list the number of discriminations out of each set of ten graphs.

\section{COMPUTATIONAL RESULTS}

\subsection{Discrimination results for regular graphs}

Tables 4.1 (a) and (b) show the results obtained with functions $\boldsymbol{w} 3, \boldsymbol{w} 4$ and $\boldsymbol{d c}$ for the regular 10 -graph sets with given $(n, d)$ values. Columns $n d i s c r$ show the number of instances (out of 10) where discrimination was obtained. Results are given for relaxed (RI) and original (OI) instances, with 1 and 4 random 2-exchanges.

These tables show $\boldsymbol{d c}(G)$ as more discriminating than $w \mathbf{4}(G)$, and $w \mathbf{4}(G)$ as more discriminating than $w 3(G)$, chiefly with lower degree values, which clearly lead to greater difficulties. Within the tests, $\boldsymbol{w} \mathbf{3}(\boldsymbol{G})$ was unable to discriminate between pairs of both 3- and 5-regular graphs, with the exception of the lesser order ones. We think this problem has to do with the absence of closed 3 -walks in the graphs. With $d>25$, or $d>50$ for greater order graphs, it produced $80 \%$ or more efficiency, while $\boldsymbol{w} \mathbf{4}(\boldsymbol{G})$ goes to $100 \%$ with $d>25$ for all cases and also for $d=10$ with lesser order graphs. 
Table 4.1(a) - Discrimination results of QAPV invariant on almost-isomorphic regular graph pairs.

\begin{tabular}{|c|c|c|c|c|c|c|c|c|c|c|c|c|c|}
\hline \multicolumn{2}{|c|}{ Instances } & \multicolumn{2}{|c|}{ One exchange } & \multicolumn{2}{|c|}{ Four exchanges } & \multicolumn{2}{|c|}{ One exchange } & \multicolumn{2}{|c|}{ Four exchanges } & \multicolumn{2}{|c|}{ One exchange } & \multicolumn{2}{|c|}{ Four exchanges } \\
\hline \multirow{2}{*}{$n$} & \multirow{2}{*}{$d$} & RI & $\mathrm{OI}$ & $\mathrm{RI}$ & OI & $\mathrm{RI}$ & OI & $\mathrm{RI}$ & OI & $\mathrm{RI}$ & OI & $\mathrm{RI}$ & OI \\
\hline & & \multicolumn{4}{|c|}{ ndiscr, Function $w 3$} & \multicolumn{4}{|c|}{ ndiscr, Function $w 4$} & \multicolumn{4}{|c|}{ ndiscr, Function $d c$} \\
\hline \multirow{4}{*}{50} & 3 & 2 & 2 & 2 & 2 & 3 & 3 & 8 & 8 & 10 & 10 & 10 & 10 \\
\hline & 5 & 5 & 5 & 9 & 10 & 9 & 10 & 10 & 10 & 10 & 10 & 10 & 10 \\
\hline & 10 & 10 & 10 & 10 & 10 & 10 & 10 & 10 & 10 & 10 & 10 & 10 & 10 \\
\hline & 25 & 9 & 10 & 10 & 10 & 10 & 10 & 10 & 10 & 9 & 9 & 9 & 9 \\
\hline \multirow{5}{*}{100} & 3 & 1 & 1 & 1 & 1 & 1 & 1 & 3 & 3 & 10 & 10 & 10 & 10 \\
\hline & 5 & 4 & 4 & 9 & 9 & 8 & 8 & 10 & 10 & 10 & 10 & 10 & 10 \\
\hline & 10 & 9 & 9 & 10 & 10 & 10 & 10 & 10 & 10 & 10 & 10 & 10 & 10 \\
\hline & 25 & 9 & 10 & 10 & 10 & 10 & 10 & 10 & 10 & 10 & 10 & 10 & 10 \\
\hline & 50 & 10 & 10 & 10 & 10 & 10 & 10 & 10 & 10 & 9 & 9 & 9 & 9 \\
\hline \multirow{6}{*}{200} & 3 & 1 & 1 & 1 & 1 & 1 & 1 & 3 & 3 & 10 & 10 & 10 & 10 \\
\hline & 5 & 1 & 1 & 2 & 2 & 6 & 7 & 10 & 10 & 10 & 10 & 10 & 10 \\
\hline & 10 & 7 & 9 & 9 & 10 & 10 & 10 & 10 & 10 & 10 & 10 & 10 & 10 \\
\hline & 25 & 10 & 10 & 10 & 10 & 10 & 10 & 10 & 10 & 10 & 10 & 10 & 10 \\
\hline & 50 & 10 & 10 & 10 & 10 & 10 & 10 & 10 & 10 & 9 & 9 & 9 & 9 \\
\hline & 100 & 10 & 10 & 10 & 10 & 10 & 10 & 10 & 10 & 9 & 9 & 9 & 9 \\
\hline \multirow{7}{*}{500} & 3 & 0 & 0 & 0 & 0 & 0 & 0 & 1 & 1 & 10 & 10 & 10 & 10 \\
\hline & 5 & 0 & 0 & 4 & 4 & 6 & 6 & 5 & 6 & 10 & 10 & 10 & 10 \\
\hline & 10 & 7 & 9 & 9 & 10 & 10 & 10 & 10 & 10 & 10 & 10 & 10 & 10 \\
\hline & 25 & 10 & 10 & 10 & 10 & 10 & 10 & 10 & 10 & 10 & 10 & 10 & 10 \\
\hline & 50 & 10 & 10 & 10 & 10 & 10 & 10 & 10 & 10 & 10 & 10 & 10 & 10 \\
\hline & 100 & 10 & 10 & 10 & 10 & 10 & 10 & 10 & 10 & 9 & 9 & 9 & 9 \\
\hline & 200 & 10 & 10 & 10 & 10 & 9 & 10 & 10 & 10 & 8 & 8 & 9 & 9 \\
\hline \multirow{8}{*}{1000} & 3 & 0 & 0 & 0 & 0 & 0 & 0 & 1 & 1 & 10 & 10 & 10 & 10 \\
\hline & 5 & 1 & 1 & 3 & 3 & 1 & 1 & 6 & 6 & 10 & 10 & 10 & 10 \\
\hline & 10 & 2 & 3 & 3 & 4 & 9 & 9 & 10 & 10 & 10 & 10 & 10 & 10 \\
\hline & 25 & 9 & 9 & 10 & 10 & 10 & 10 & 10 & 10 & 10 & 10 & 10 & 10 \\
\hline & 50 & 10 & 10 & 10 & 10 & 10 & 10 & 10 & 10 & 10 & 10 & 10 & 10 \\
\hline & 100 & 10 & 10 & 10 & 10 & 10 & 10 & 10 & 10 & 2 & 3 & 2 & 7 \\
\hline & 200 & 10 & 10 & 10 & 10 & 10 & 10 & 10 & 10 & 1 & 9 & 1 & 9 \\
\hline & 500 & 10 & 10 & 10 & 10 & 10 & 10 & 10 & 10 & - & - & - & - \\
\hline
\end{tabular}

Function $\boldsymbol{d c}(\boldsymbol{G})$ was $100 \%$ efficient with all instance sets of lower degree, thus complementing the range of the other two functions. We can see that it does not perform as well with the denser graphs, owing probably to the equalization of the number of centers and to lesser distance ranges. The results from the $(1000,100)$ and $(1000,200)$ pairs, for instance, were not good and that led us to abandon the idea of testing higher degrees.

We can also observe that, in most cases, a single 2-exchange was enough for the invariant to show a structural difference, specially with function $\boldsymbol{d c}(\boldsymbol{G})$.

Finally, we exemplify the application of $\operatorname{es}(w 3(G))$ and $\operatorname{es}(w 4(G))$ by using it with lesser-degree instances, where $\boldsymbol{w} \mathbf{3}(G)$, and even $\boldsymbol{w} \mathbf{4}(G)$, encountered difficulties in discriminating between pairs of almost isomorphs. Table 4.2 shows these results, which can be compared with those from Tables 4.1 (a) and (b) for the same instance collections.

We put in bold type the $n$ discr values that were enhanced by the use of es $(f(G))$ when compared with the original values of $\boldsymbol{w} \mathbf{3}(\boldsymbol{G})$ and $\boldsymbol{w} \mathbf{4}(\boldsymbol{G})$ (Tables 4.1 (a) and (b)). We can see that they 
Table 4.1(b) - Discrimination results of QAPV invariant on almost-isomorphic regular graph pairs.

\begin{tabular}{|c|c|c|c|c|c|c|c|c|c|c|c|c|c|}
\hline \multicolumn{2}{|c|}{ Instances } & \multicolumn{2}{|c|}{ One exchange } & \multicolumn{2}{|c|}{ Four exchanges } & \multicolumn{2}{|c|}{ One exchange } & \multicolumn{2}{|c|}{ Four exchanges } & \multicolumn{2}{|c|}{ One exchange } & \multicolumn{2}{|c|}{ Four exchanges } \\
\hline \multirow{2}{*}{$n$} & \multirow{2}{*}{$d$} & RI & OI & RI & OI & RI & OI & RI & OI & RI & OI & RI & OI \\
\hline & & \multicolumn{4}{|c|}{ ndiscr, Function $w 3$} & \multicolumn{4}{|c|}{ ndiscr, Function $w 4$} & \multicolumn{4}{|c|}{$n d i s c r$, Function $d c$} \\
\hline \multirow{7}{*}{1500} & 3 & 0 & 0 & 0 & 0 & 0 & 0 & 0 & 0 & 10 & 10 & 10 & 10 \\
\hline & 5 & 0 & 0 & 2 & 2 & 0 & 0 & 3 & 4 & 10 & 10 & 10 & 10 \\
\hline & 10 & 3 & 3 & 5 & 5 & 9 & 9 & 8 & 8 & 10 & 10 & 10 & 10 \\
\hline & 25 & 7 & 8 & 9 & 9 & 10 & 10 & 10 & 10 & 10 & 10 & 10 & 10 \\
\hline & 50 & 10 & 10 & 10 & 10 & 10 & 10 & 10 & 10 & 10 & 10 & 10 & 10 \\
\hline & 100 & 10 & 10 & 10 & 10 & 10 & 10 & 10 & 10 & 2 & 9 & 10 & 10 \\
\hline & 200 & 10 & 10 & 10 & 10 & 10 & 10 & 10 & 10 & - & - & - & - \\
\hline \multirow{7}{*}{2000} & 3 & 0 & 0 & 0 & 0 & 0 & 0 & 0 & 0 & 10 & 10 & 10 & 10 \\
\hline & 5 & 0 & 0 & 5 & 5 & 0 & 0 & 3 & 3 & 10 & 10 & 10 & 10 \\
\hline & 10 & 3 & 3 & 5 & 5 & 6 & 6 & 10 & 10 & 10 & 10 & 10 & 10 \\
\hline & 25 & 6 & 6 & 9 & 10 & 10 & 10 & 10 & 10 & 10 & 10 & 10 & 10 \\
\hline & 50 & 10 & 10 & 10 & 10 & 10 & 10 & 10 & 10 & - & - & - & - \\
\hline & 100 & 10 & 10 & 10 & 10 & 10 & 10 & 10 & 10 & - & - & - & - \\
\hline & 200 & 10 & 10 & 10 & 10 & - & - & - & - & - & - & - & - \\
\hline \multirow{7}{*}{2500} & 3 & 0 & 0 & 0 & 0 & 0 & 0 & 0 & 0 & 10 & 10 & 10 & 10 \\
\hline & 5 & 0 & 0 & 0 & 0 & 0 & 0 & 2 & 2 & 10 & 10 & 10 & 10 \\
\hline & 10 & 2 & 2 & 4 & 4 & 6 & 6 & 9 & 9 & 10 & 10 & 10 & 10 \\
\hline & 25 & 5 & 5 & 10 & 10 & 10 & 10 & 10 & 10 & 10 & 10 & 10 & 10 \\
\hline & 50 & 10 & 10 & 10 & 10 & 10 & 10 & 10 & 10 & - & - & - & - \\
\hline & 100 & 10 & 10 & 10 & 10 & 10 & 10 & 10 & 10 & - & - & - & - \\
\hline & 200 & 10 & 10 & 10 & 10 & - & - & - & - & - & - & - & - \\
\hline \multirow{7}{*}{3000} & 3 & 0 & 0 & 0 & 0 & 0 & 0 & 0 & 0 & 10 & 10 & 10 & 10 \\
\hline & 5 & 0 & 0 & 1 & 1 & 1 & 1 & 2 & 2 & 10 & 10 & 10 & 10 \\
\hline & 10 & 0 & 0 & 3 & 3 & 7 & 7 & 9 & 9 & 10 & 10 & 10 & 10 \\
\hline & 25 & 6 & 7 & 9 & 9 & 10 & 10 & 10 & 10 & 10 & 10 & 10 & 10 \\
\hline & 50 & 9 & 9 & 10 & 10 & 10 & 10 & 10 & 10 & - & - & - & - \\
\hline & 100 & 10 & 10 & 10 & 10 & - & - & - & - & - & - & - & - \\
\hline & 200 & 10 & 10 & 10 & 10 & - & - & - & - & - & - & - & - \\
\hline
\end{tabular}

constitute a significant majority of those values that can be enhanced (discr $<10$ ), (see, for instance, the 25-regular graphs with $\boldsymbol{w} 4(\boldsymbol{G}), n \geq 1500$, compared with those of Table 4.1(b)). In the whole, this experiment shows about $78 \%$ of enhanced values, which is interesting because of the very low cost of this function. For some instances, this application is fruitless; some examples are the 3-regular graphs with 1000 or more vertices. We think that, in these cases, the structures associated with $w \mathbf{3}(G)$ and $w \mathbf{4}(G)$ (3- and 4-closed walks) do not easily exist in the graphs, a situation which can be found in large, low-degree regular graphs, where larger girths are to be expected.

\subsection{Processing times for regular graphs}

Processing times are based on the use of a computer with an Intel Core i7 / $9753.33 \mathrm{Ghz}$ processor, with $8 \mathrm{~Gb}$ of RAM, operating with Linux OpenSUSE 11.2 and a 64-bit Intel Fortran compiler. Figure 4.1 shows the weight functions' processing times for the instances presented in the preceding tables. 
Table 4.2 - Application of es $(f(G))$ to some instance collections affected by $w 3(G)$ and $w 4(G)$.

\begin{tabular}{|c|c|c|c|c|c|c|c|c|c|}
\hline \multicolumn{2}{|c|}{ Graph } & \multicolumn{2}{|c|}{ One exchange } & \multicolumn{2}{|c|}{ Four exchanges } & \multicolumn{2}{|c|}{ One exchange } & \multicolumn{2}{|c|}{ Four exchanges } \\
\hline \multirow{2}{*}{$n$} & \multirow{2}{*}{$d$} & RI & OI & RI & OI & RI & OI & RI & OI \\
\hline & & \multicolumn{4}{|c|}{ ndiscr, Function $w 3$} & \multicolumn{4}{|c|}{ ndiscr, Function $w 4$} \\
\hline \multirow{2}{*}{50} & 3 & 3 & 6 & 4 & 5 & 4 & 8 & 10 & 10 \\
\hline & 5 & 7 & 7 & 10 & 10 & 10 & 10 & 10 & 10 \\
\hline \multirow{2}{*}{100} & 3 & 1 & 1 & 1 & 1 & 4 & 8 & 4 & 10 \\
\hline & 5 & 6 & 6 & 9 & 9 & 10 & 10 & 10 & 10 \\
\hline \multirow{3}{*}{200} & 3 & 2 & 2 & 1 & 1 & 2 & 4 & 6 & 9 \\
\hline & 5 & 1 & 1 & 5 & 7 & 7 & 10 & 10 & 10 \\
\hline & 10 & 9 & 10 & 10 & 10 & 10 & 10 & 10 & 10 \\
\hline \multirow{3}{*}{500} & 3 & 0 & 0 & 0 & 1 & 3 & 5 & 6 & 8 \\
\hline & 5 & 1 & 1 & 5 & 7 & 8 & 9 & 10 & 10 \\
\hline & 10 & 8 & 8 & 9 & 10 & 10 & 10 & 10 & 10 \\
\hline \multirow{3}{*}{1000} & 3 & 0 & 0 & 1 & 1 & 4 & 5 & 4 & 9 \\
\hline & 5 & 2 & 4 & 7 & 8 & 5 & 7 & 10 & 10 \\
\hline & 10 & 4 & 7 & 4 & 8 & 9 & 9 & 10 & 10 \\
\hline \multirow{4}{*}{1500} & 3 & 0 & 0 & 0 & 0 & 1 & 5 & 7 & 8 \\
\hline & 5 & 1 & 1 & 3 & 4 & 1 & 5 & 8 & 10 \\
\hline & 10 & 4 & 5 & 7 & 9 & 9 & 9 & 10 & 10 \\
\hline & 25 & 7 & 9 & 10 & 10 & 10 & 10 & 10 & 10 \\
\hline \multirow{4}{*}{2000} & 3 & 0 & 0 & 0 & 0 & 3 & 4 & 3 & 7 \\
\hline & 5 & 1 & 1 & 6 & 8 & 2 & 5 & 7 & 9 \\
\hline & 10 & 4 & 5 & 5 & 6 & 9 & 9 & 10 & 10 \\
\hline & 25 & 7 & 10 & 10 & 10 & 10 & 10 & 10 & 10 \\
\hline \multirow{4}{*}{2500} & 3 & 0 & 1 & 1 & 1 & 4 & 4 & 5 & 8 \\
\hline & 5 & 1 & 2 & 0 & 2 & 5 & 7 & 7 & 10 \\
\hline & 10 & 3 & 3 & 6 & 8 & 8 & 8 & 10 & 10 \\
\hline & 25 & 5 & 6 & 10 & 10 & 10 & 10 & 10 & 10 \\
\hline \multirow{4}{*}{3000} & 3 & 0 & 0 & 0 & 0 & 2 & 6 & 3 & 9 \\
\hline & 5 & 0 & 2 & 2 & 4 & 3 & 5 & 8 & 9 \\
\hline & 10 & 1 & 1 & 5 & 5 & 8 & 8 & 10 & 10 \\
\hline & 25 & 6 & 8 & 9 & 10 & 10 & 10 & 10 & 10 \\
\hline
\end{tabular}

We can see that the processing time for es(.) is negligible when compared with the other functions.

By examining Figures 4.1 and 4.2, we see that the graphs show comparable times between $\boldsymbol{w} 3(\boldsymbol{G})$ and variance calculation, with $\boldsymbol{w} \mathbf{4}(G)$ taking more time. The times depend on the graph degree with the exception of $\boldsymbol{d c}(\boldsymbol{G})$, whose time requirements are almost constant for a given order.

\subsection{Going to practical application}

The following conjecture, which we did not attempt to prove, is supported by our tests: 


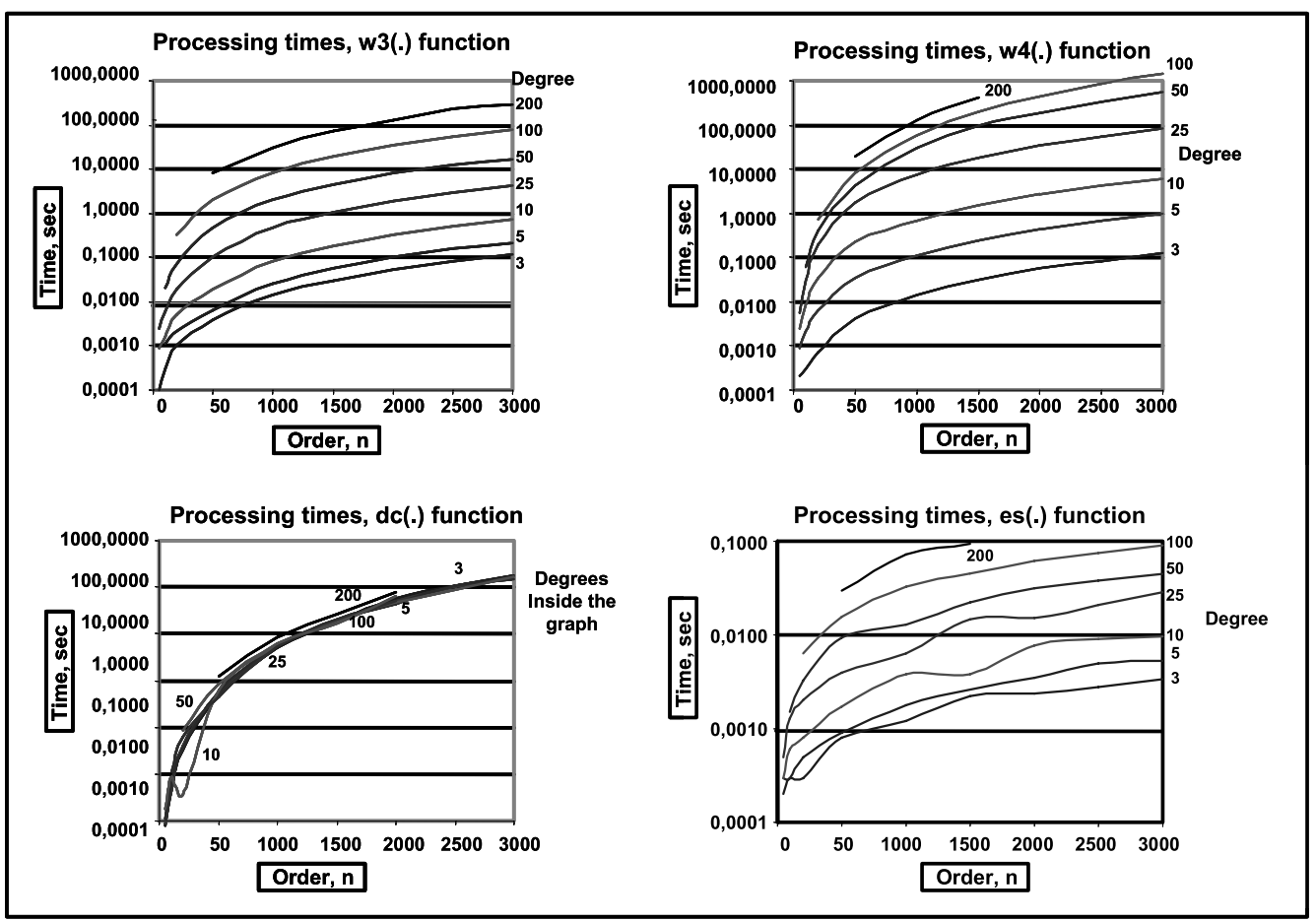

Figure 4.1 - Processing times for weight functions applied to regular graphs.

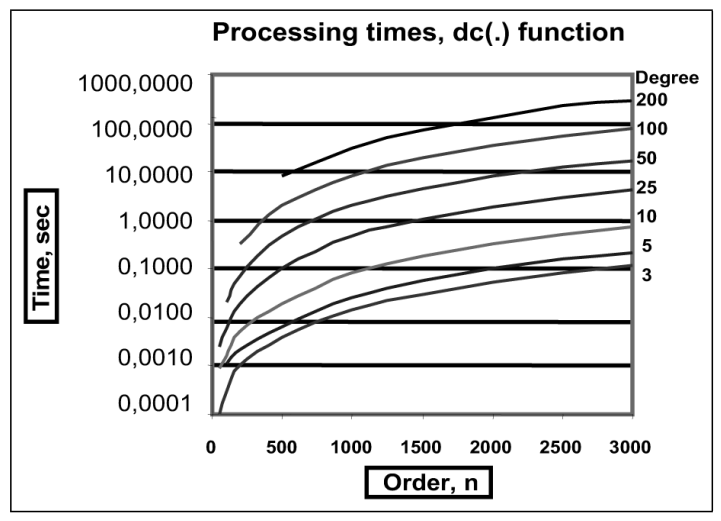

Figure 4.2 - Processing times of QAPV invariant for regular QAP-GIP instances.

Conjecture 4.1. For every graph pair $\left(G_{1}, G_{2}\right)$ of the same order and size, there exists a polynomial invariant weight function such that, for non-isomorphic $G_{1}$ and $G_{2}$, the instances $Q A P\left(G_{1}, G_{2}\right), Q A P\left(G_{1}, G_{1}\right)$ and $Q A P\left(G_{2}, G_{2}\right)$ will have different variances.

We summarize some tests done with the three functions $w \mathbf{3}(G), w \mathbf{4}(G)$ and $d c(G)$ for some orderdegree pairs and one 2-exchange in order to show that, for every pair $(n, d)$, we always have a function providing $100 \%$ discrimination among the almost-isomorphic pairs. We outline the computing time requirements as shown by Table 4.3, where they are expressed in seconds. 
Table 4.3 - Discrimination and total QAPV processing time for some regular graph instances.

\begin{tabular}{|c|c|c|c|c|c|}
\hline $\begin{array}{c}\text { Order } \\
n\end{array}$ & $\begin{array}{c}\text { Degree } \\
d\end{array}$ & $\begin{array}{l}\text { Function time } \\
\qquad \begin{array}{c}w 3(G) \\
w 4(G) \\
d c(G)\end{array}\end{array}$ & $\begin{array}{c}\text { Variance } \\
\text { time }\end{array}$ & $\begin{array}{l}\text { Total } \\
\text { time }\end{array}$ & Discr.\% \\
\hline \multirow{6}{*}{50} & \multirow{3}{*}{3} & 0.0002 & 0,0005 & 0.0007 & 20 \\
\hline & & 0.0004 & 0,0005 & 0.0009 & $30-80$ \\
\hline & & 0.0016 & 0.0005 & 0.0021 & 100 \\
\hline & \multirow{3}{*}{25} & 0.0048 & 0.0024 & 0.0072 & $90-100$ \\
\hline & & 0.0110 & 0.0024 & 0.0134 & 100 \\
\hline & & 0.0036 & 0.0024 & 0.0060 & 90 \\
\hline \multirow{6}{*}{200} & \multirow{3}{*}{5} & 0.0042 & 0.0016 & 0.0058 & $10-20$ \\
\hline & & 0.0128 & 0.0016 & 0.0144 & $60-100$ \\
\hline & & 0.0780 & 0.0016 & 0.0796 & 100 \\
\hline & \multirow{3}{*}{50} & 0.1410 & 0.1025 & 0.2435 & 100 \\
\hline & & 0.7980 & 0.1025 & 0.9005 & 100 \\
\hline & & 0.1270 & 0.1025 & 0.2295 & 90 \\
\hline \multirow{6}{*}{500} & \multirow{3}{*}{10} & 0.039 & 0.027 & 0.066 & 80 \\
\hline & & 0.440 & 0.027 & 0.467 & 100 \\
\hline & & 1.140 & 0.027 & 1.167 & 100 \\
\hline & \multirow{3}{*}{100} & 3.97 & 2.34 & 6.31 & 100 \\
\hline & & 16.31 & 2.341 & 8.65 & 100 \\
\hline & & 1.73 & 2.34 & 4.07 & 90 \\
\hline \multirow{6}{*}{1000} & \multirow{3}{*}{10} & 0.08 & 0.96 & 1.04 & $20-40$ \\
\hline & & 0.67 & 0.96 & 1.63 & $90-100$ \\
\hline & & 5.13 & 0.96 & 6.09 & 100 \\
\hline & \multirow{3}{*}{100} & 8.03 & 93.76 & 101.79 & 100 \\
\hline & & 59.00 & 93.76 & 152.76 & 100 \\
\hline & & 5.97 & 93.76 & 99.73 & $20-70$ \\
\hline \multirow{9}{*}{1500} & \multirow{3}{*}{10} & 0.19 & 2.15 & 2.34 & $30-50$ \\
\hline & & 1.52 & 2.15 & 3.67 & $80-90$ \\
\hline & & 18.83 & 2.15 & 20.98 & 100 \\
\hline & \multirow{3}{*}{25} & 1.08 & 13.32 & 14.40 & $70-90$ \\
\hline & & 18.51 & 13.32 & 29.83 & 100 \\
\hline & & 19.75 & 13.32 & 33.07 & 100 \\
\hline & \multirow{3}{*}{100} & 18.37 & 21.04 & 39.41 & 100 \\
\hline & & 191.83 & 21.04 & 212.87 & 100 \\
\hline & & 16.54 & 21.04 & 37.94 & $20-100$ \\
\hline \multirow{6}{*}{2000} & \multirow{3}{*}{10} & 0.66 & 0.38 & 1.04 & $30-50$ \\
\hline & & 5.39 & 0.38 & 5.77 & $60-100$ \\
\hline & & 90.78 & 0.38 & 91.16 & 100 \\
\hline & \multirow{3}{*}{25} & 3.80 & 2.35 & 6.15 & $60-100$ \\
\hline & & 68.76 & 2.35 & 71.11 & 100 \\
\hline & & 93.94 & 2.35 & 96.29 & 100 \\
\hline \multirow{3}{*}{3000} & \multirow{3}{*}{25} & 8.50 & 5.27 & 13.77 & $60-90$ \\
\hline & & 161.66 & 5.27 & 166.93 & 100 \\
\hline & & 330.30 & 5.27 & 335.57 & 100 \\
\hline
\end{tabular}




\section{TESTS WITH TRIANGULATED-GRID GRAPHS}

The almost-isomorphs used in the tests allowed $100 \%$ discrimination with the $(50,80),(50,100)$, $(70,100),(100,100),(150,100),(200,100),(200,200)$ and $(500,500)$ instances. The $(200,150)$, $(250,200),(300,200),(400,200),(400,250),(500,300)$ and $(500,400)$ instances had $90 \%$ discrimination. The $(600,500)$ instance testes showed $70 \%$ discrimination. Discrimination was always the same with relaxed and original instances. For each of these tests, five almost-isomorphs were randomly generated and compared two by two, thus allowing ten comparisons.

The processing time of all relaxed instances was negligible $(\leq 0.002 \mathrm{sec})$. Figure 5.1 shows the processing time for the original instances.

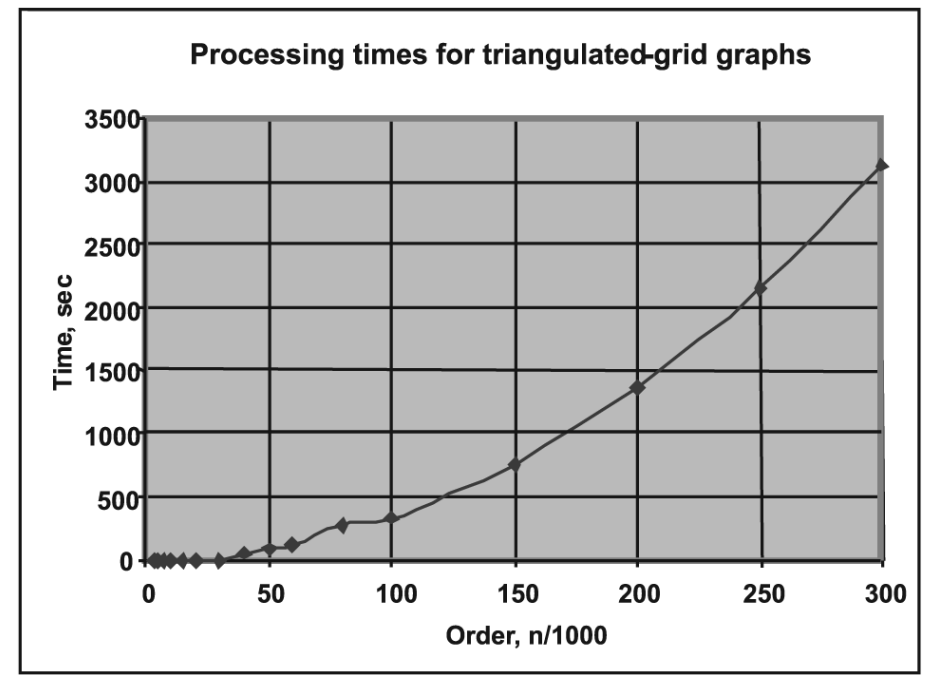

Figure 5.1 - Processing times of QAPV for TGG.

As an example, Figure 5.2 shows a $(15,11)$-TGG with four windows (indicated by the circles).

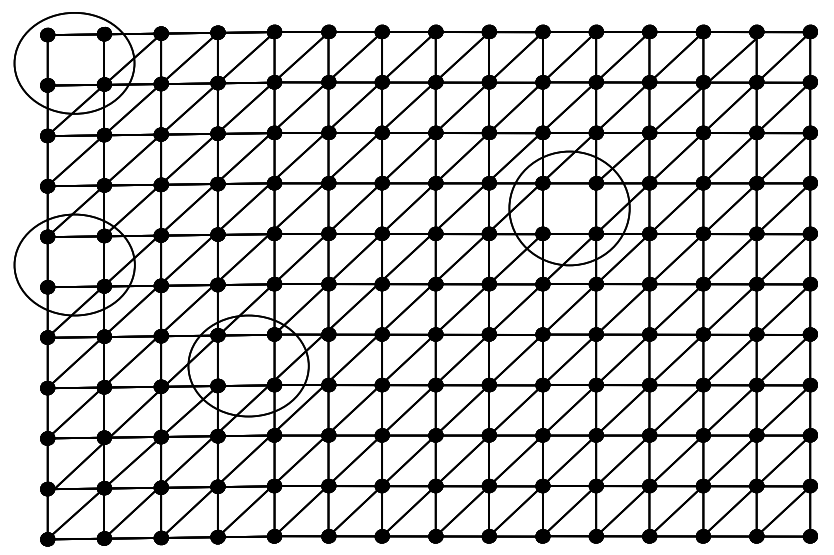

Figure 5.2 - A (15,11)-TGG with four windows. 


\section{AN EXAMPLE OF ISOMORPHISM}

As a case of isomorphism, we present a $(30,8)$ graph and an isomorph of it characterized by a given permutation, applying $\boldsymbol{w} \mathbf{3}(G)$ to both of them. The graphs are described by their nonsymmetric valued adjacency lists, firstly $v_{i} \in V$, then $v_{j} \mid \exists\left(v_{i}, v_{j}\right), j>i$ and finally, the corresponding $\boldsymbol{w} 3(G)$ edge values. The main instance and its associates produced the same variance values (R\% null), both with the relaxed and with the original instances (Fig. 6.1):

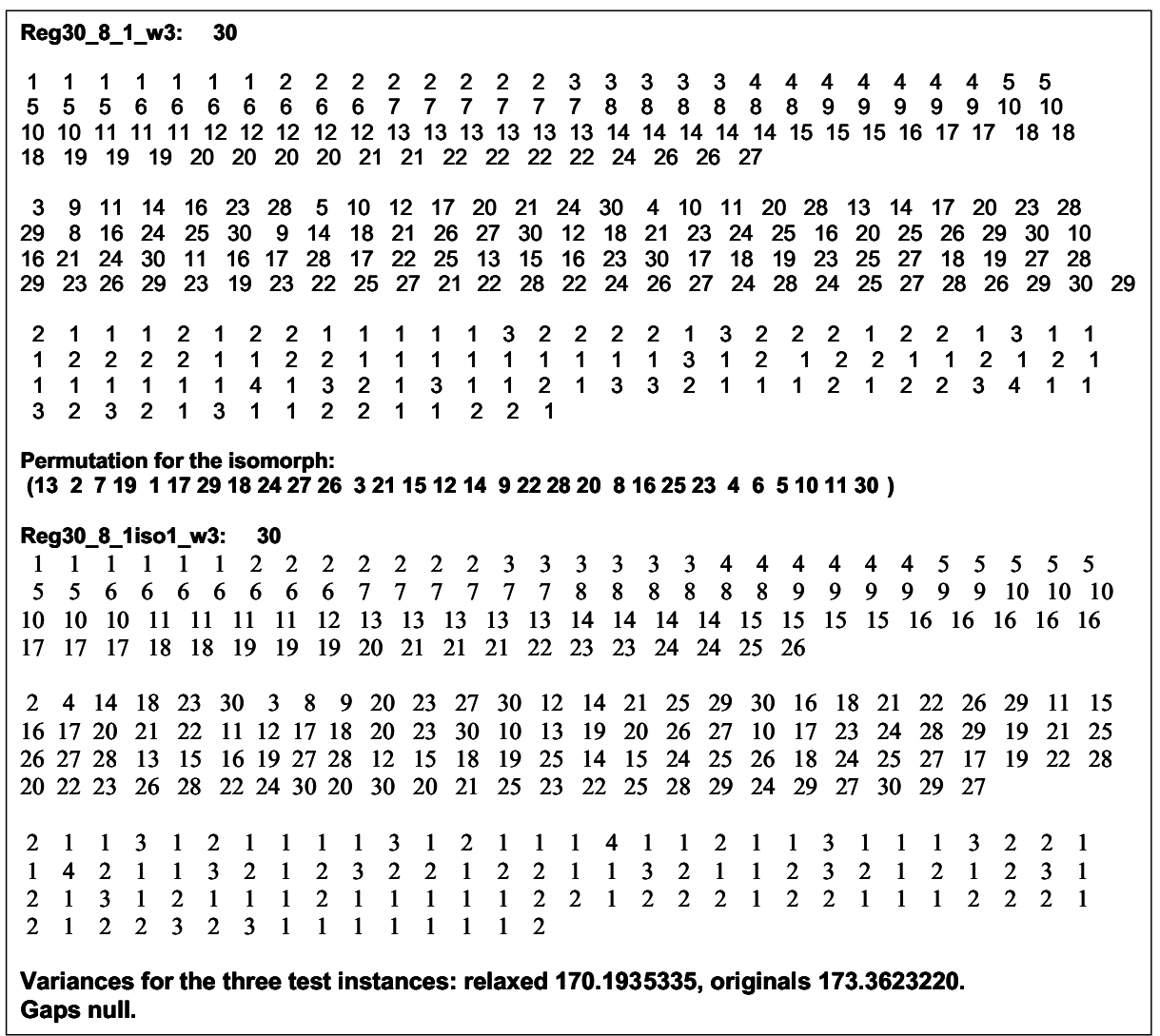

Figure 6.1 - A regular graph and one isomorph.

\section{LOOKING FOR A COMPARISON}

7.1 The most recent results on graph isomorphism refer to progressive assembly of structures, either using exact algorithms or with the aid of heuristics The exact algorithms are often highly complex: its elaborate construction allows their use with pairs of graphs of order relatively high (e.g., 500 vertices) but the processing time varies greatly according to the permutation found. Heuristics have higher possibilities of approach and can be useful, for example, in applications of pattern recognition, but their result is prone to error. All these techniques are iterative, unlike the invariant calculations, which do not involve alternate returns as seen in Figure 2.1. 
Anyway, we try to present some comparisons, involving two aspects:

7.1.1 The calculation of QAPV invariant for public access graphs collections on the Internet.

1. We used the first ten graph pairs of the sets $\mathbf{r 0 0 1}, \boldsymbol{r 0 0 5}$ and $\boldsymbol{r 0 1}$, with orders $20 \leq n \leq$ 600 , indicated as isomorphs in the category randomly connected graphs, from Santo et al. [32] database. We also processed pairs indicated as $\mathrm{xxA} / \mathrm{xxB} 1 \mathrm{ex}$ and $\mathrm{xxA} 1 \mathrm{ex} / \mathrm{xxB}$ (where the suffix 1ex indicates almost-isomorphic with one 2-exchange), obtained from the corresponding $\mathrm{xxA}$ and $\mathrm{xxB}$ original graphs.

2. We generated almost-isomorphs with one 2-exchange for two sets of 19 strongly regular graphs [20] of 45 and 64 vertices (numbered 001-019), each graph being tested along with its almost-isomorph. The invariant obtained discrimination in all cases, using the functions $w 3(G)$ and $w 4(G)$, the $d c(G)$ function was not efficient in these cases.

Table 7.1 indicates the number of pairs (out of 10) from Test 1, for which there was discrimination, both for the relaxed instances (RI) and the original ones (OI). The pairs (A, B) are the original graph pairs, while the suffix "1ex." indicates one 2-exchange in A or B file. There was no discrimination between the original pairs.

Table 7.1 - Results obtained with Santo et al. database.

\begin{tabular}{|c|c|c|c|c|c|c|c|c|c|c|c|c|c|c|c|c|c|c|c|c|c|c|c|c|c|}
\hline \multirow{3}{*}{\multicolumn{2}{|c|}{$\begin{array}{l}\text { Instance } \\
\text { collection }\end{array}$}} & \multicolumn{6}{|c|}{ Function: es $(G)$} & \multicolumn{6}{|c|}{ Function: $w 3(G)$} & \multicolumn{6}{|c|}{ Function: $w 4(G)$} & \multicolumn{6}{|c|}{ Function: $d c(G)$} \\
\hline & & \multicolumn{2}{|c|}{$(\mathrm{A}, \mathrm{B})$} & \multicolumn{2}{|c|}{ A1ex. } & \multicolumn{2}{|c|}{ B1ex. } & \multicolumn{2}{|c|}{$(\mathrm{A}, \mathrm{B})$} & \multicolumn{2}{|c|}{ A1ex. } & \multicolumn{2}{|c|}{ B1ex. } & \multicolumn{2}{|c|}{$(\mathrm{A}, \mathrm{B})$} & \multicolumn{2}{|c|}{ A1ex. } & \multicolumn{2}{|c|}{ B1ex. } & \multicolumn{2}{|c|}{$(\mathrm{A}, \mathrm{B})$} & \multicolumn{2}{|c|}{ Alex. } & \multicolumn{2}{|c|}{ B1ex. } \\
\hline & & RI & OI & RI & OI & RI & OI & RI & $\mathrm{OI}$ & RI & OI & RI & OI & RI & OI & RI & OI & RI & OI & RI & OI & RI & OI & RI & OI \\
\hline \multirow[t]{8}{*}{$\mathrm{r} 001$} & 0020 & 0 & 0 & 2 & 2 & 0 & 0 & 0 & 0 & 10 & 10 & 10 & 10 & 0 & 0 & 1 & 1 & 3 & 4 & 0 & 0 & 10 & 10 & 10 & 10 \\
\hline & 0040 & 0 & 0 & 0 & 0 & 0 & 0 & 0 & 0 & 10 & 10 & 10 & 10 & 0 & 0 & 3 & 3 & 2 & 2 & 0 & 0 & 8 & 8 & 8 & 8 \\
\hline & 0060 & 0 & 0 & 6 & 9 & 7 & 7 & 0 & 0 & 10 & 10 & 10 & 10 & 0 & 0 & 6 & 9 & 7 & 7 & 0 & 0 & 7 & 7 & 7 & 7 \\
\hline & 0080 & 0 & 0 & 6 & 10 & 10 & 10 & 0 & 0 & 10 & 10 & 10 & 10 & 0 & 0 & 7 & 10 & 10 & 10 & 0 & 0 & 10 & 10 & 10 & 10 \\
\hline & 0100 & 0 & 0 & 4 & 9 & 5 & 9 & 0 & 0 & 10 & 10 & 10 & 10 & 0 & 0 & 8 & 10 & 6 & 9 & 0 & 0 & 10 & 10 & 9 & 9 \\
\hline & 0200 & 0 & 0 & 7 & 10 & 9 & 10 & 0 & 0 & 10 & 10 & 10 & 10 & 0 & 0 & 9 & 10 & 9 & 10 & 0 & 0 & 10 & 10 & 10 & 10 \\
\hline & 0400 & 0 & 0 & 7 & 10 & 4 & 9 & 0 & 0 & 10 & 10 & 10 & 10 & 0 & 0 & 10 & 10 & 10 & 10 & 0 & 0 & 10 & 10 & 10 & 10 \\
\hline & 0600 & 0 & 0 & 9 & 10 & 10 & 10 & 0 & 0 & 10 & 10 & 10 & 10 & 0 & 0 & 10 & 10 & 10 & 10 & 0 & 0 & 10 & 10 & 9 & 10 \\
\hline \multirow[t]{8}{*}{ r005 } & 0020 & 0 & 0 & 5 & 8 & 7 & 10 & 0 & 0 & 4 & 4 & 3 & 3 & 0 & 0 & 6 & 8 & 9 & 10 & 0 & 0 & 8 & 9 & 10 & 10 \\
\hline & 0040 & 0 & 0 & 9 & 10 & 7 & 10 & 0 & 0 & 4 & 6 & 5 & 5 & 0 & 0 & 10 & 10 & 10 & 10 & 0 & 0 & 7 & 10 & & 10 \\
\hline & 0060 & 0 & 0 & 9 & 10 & 7 & 10 & 0 & 0 & 8 & 9 & 7 & 8 & 0 & 0 & 10 & 10 & 10 & 10 & 0 & 0 & 10 & 10 & 10 & 10 \\
\hline & 0080 & 0 & 0 & 8 & $\overline{9}$ & 9 & 10 & 0 & 0 & 8 & 9 & 7 & 8 & 0 & 0 & 10 & 10 & 10 & 10 & 0 & 0 & 10 & 10 & 10 & 10 \\
\hline & 0100 & 0 & 0 & 7 & 9 & 7 & 10 & 0 & 0 & 9 & 9 & 9 & 10 & 0 & 0 & 10 & 10 & 10 & 10 & 0 & 0 & 10 & 10 & 10 & 10 \\
\hline & 0200 & 0 & 0 & 10 & 10 & 7 & 10 & 0 & 0 & 10 & 10 & 10 & 10 & 0 & 0 & 10 & 10 & 10 & 10 & 0 & 0 & 10 & 10 & 10 & 10 \\
\hline & 0400 & 0 & 0 & 9 & 10 & 8 & 10 & 0 & 0 & 10 & 10 & 10 & 10 & 0 & 0 & 10 & 10 & 10 & 10 & 0 & 0 & 6 & 8 & 10 & 8 \\
\hline & 0600 & 0 & 0 & 10 & 10 & 10 & 10 & 0 & 0 & 10 & 10 & 10 & 10 & 0 & 0 & 10 & 10 & 10 & 10 & 0 & 0 & 10 & 10 & 10 & 10 \\
\hline \multirow[t]{8}{*}{$\mathrm{r} 01$} & 0020 & 0 & 0 & 5 & 7 & 6 & 8 & 0 & 0 & 10 & 10 & 10 & 10 & 0 & 0 & 9 & 9 & 10 & 10 & 0 & 0 & 10 & 10 & 10 & 10 \\
\hline & 0040 & 0 & 0 & 8 & 10 & 6 & 10 & 0 & 0 & 10 & 10 & 10 & 10 & 0 & 0 & 10 & 10 & 10 & 10 & 0 & 0 & 6 & 8 & 6 & 9 \\
\hline & 0060 & 0 & 0 & 9 & 10 & 8 & 9 & 0 & 0 & 10 & 10 & 9 & 10 & 0 & 0 & 10 & 10 & 10 & 10 & 0 & 0 & 5 & 7 & 5 & 6 \\
\hline & 0080 & 0 & 0 & 9 & 10 & 9 & 10 & 0 & 0 & 9 & 10 & 9 & 10 & 0 & 0 & 10 & 10 & 10 & 10 & 0 & 0 & 10 & 10 & 6 & 9 \\
\hline & 0100 & 0 & 0 & 10 & 10 & 8 & 10 & 0 & 0 & 10 & 10 & 10 & 10 & 0 & 0 & 10 & 10 & 10 & 10 & 0 & 0 & 10 & 10 & 6 & 9 \\
\hline & 0200 & 0 & 0 & 10 & 10 & 10 & 10 & 0 & 0 & 10 & 10 & 10 & 10 & 0 & 0 & 10 & 10 & 10 & 10 & 0 & 0 & 7 & 10 & 9 & 10 \\
\hline & 0400 & 0 & 0 & 9 & 10 & 9 & 9 & 0 & 0 & 10 & 10 & 10 & 10 & 0 & 0 & 10 & 10 & 10 & 10 & 0 & 0 & 9 & 9 & 9 & 9 \\
\hline & 0600 & 0 & 0 & 9 & 10 & 9 & 10 & 0 & 0 & 10 & 10 & 10 & 10 & 0 & 0 & 10 & 10 & 10 & 10 & 0 & 0 & 9 & 9 & 9 & 9 \\
\hline
\end{tabular}


The results from Test 2, with strongly regular graphs and their almost-isomorphs, are indicated in Table 7.2:

Table 7.2 - Results for strongly regular graphs.

\begin{tabular}{|c|c|c|c|c|c|c|}
\hline \multirow{2}{*}{ Instance } & \multicolumn{2}{|c|}{$w 3(G)$} & \multicolumn{2}{c|}{$w 4(G)$} & \multicolumn{2}{c|}{$d c(G)$} \\
\cline { 2 - 7 } & RI & OI & RI & OI & RI & OI \\
\hline$(45,12,3,3)$ & 19 & 19 & 19 & 19 & 0 & 0 \\
$(64,18,2,6)$ & 19 & 19 & 19 & 19 & 0 & 0 \\
\hline
\end{tabular}

7.1.2 We applied the algorithm VF2 [33] to regular graphs of order 100, 200 and 500 from the database already used in this work. We created isomorphs for these graphs and formed pairs (graph, isomorph). We also used the same one 2-exchange (graph, almost-isomorph) pairs referred to in Table 4.1 (a).

For the application of VF2 to our instances we used the IGRAPH library [21], version 0.5.4, a free software package for creating and manipulating undirected and directed graphs which contains the VF2 algorithm. All instances were put in Dimacs format and submitted to VF2.

In order to compare the runtime of our algorithm with the library VF2, we needed a maximum time limit for large instances. However, IGRAPH is a closed pack which only stops when it finishes processing. To guarantee a time limit, we created two jobs (shell) using Linux OS. The first shell (JOB1.sh), controls the time limit and the second shell (JOB2.sh) uses the VF2 library in order to check all instances for isomorphism.

If $J O B 1 . s h$ finishes first, then JOB2.sh execution time is equal or greater than the time limit and $J O B 1 . s h$ interrupts both child processes. If, on the contrary, JOB2.sh finishes within the time limit, it interrupts JOB1.sh execution before stopping. See Figure 7.1.

\begin{tabular}{|l|l|}
\begin{tabular}{|l} 
JOB1.sh \\
for all instances \\
verify the existence of the instance in the current directory \\
create JOB1 control file \\
control the maximum processing time \\
$\begin{array}{l}\text { verify the existence of the control files } \\
\text { if they exist then } \\
\text { remove control files } \\
\text { kill both child processes }\end{array}$
\end{tabular} & $\begin{array}{l}\text { JOB2.sh } \\
\text { for all instances } \\
\text { verify the existence of the instance in the current directory } \\
\text { create JOB2 control file } \\
\text { execute VF2 library }\end{array}$ \\
$\begin{array}{l}\text { verify the existence of the control files } \\
\text { if they exist then }\end{array}$ & $\begin{array}{l}\text { remove control files } \\
\text { kill child process of JOB1.sh }\end{array}$ \\
end for
\end{tabular}

Figure 7.1 - Pseudocodes of JOB1 and JOB2 (shells).

Tables 7.3 and 7.4 show the results for each $(n, d)$ ten-graph collection, both for QAPV and VF2, respectively with isomorphic and almost-isomorphic pairs. The maximum processing time allowed for VF2 was 3,600 seconds. All times are expressed in seconds.

The function $\boldsymbol{d} \boldsymbol{c}(\boldsymbol{G})$ was utilized in these tests, with the exception of $(500,100)$ and $(500,200)$, since it is not very efficient in these cases (see Table 4.1(a)). We replaced it by $\boldsymbol{w} 3(\boldsymbol{G})$, its corresponding time values being written in italic. The second column shows the time average for 
Table 7.3 - QAPV-VF2 comparison of processing time with isomorphic pairs.

\begin{tabular}{|c|c|c|c|c|c|c|c|c|}
\hline Instance & \multicolumn{7}{|c|}{ QAPV } & \multicolumn{3}{c|}{ VF2 } \\
\cline { 2 - 9 } n,d & avg, dc & avg & $\Sigma$ avg & $\max$ & $\min$ & avg & $\max$ & $\min$ \\
\hline 100,3 & 0.011 & 0.001 & 0.012 & 0.020 & 0.008 & 0.158 & 0.484 & 0.001 \\
100,10 & 0.014 & 0.002 & 0.016 & 0.026 & 0.011 & 0.075 & 0.246 & 0.003 \\
100,25 & 0.017 & 0.008 & 0.025 & 0.036 & 0.021 & 0.123 & 0.448 & 0.001 \\
100,50 & 0.021 & 0.029 & 0.050 & 0.066 & 0.046 & 0.303 & 0.839 & 0.016 \\
200,3 & 0.039 & 0.001 & 0.040 & 0.050 & 0.036 & 98.903 & 472.88 & 0.002 \\
200,10 & 0.039 & 0.005 & 0.044 & 0.060 & 0.040 & 10.017 & 85,347 & 0.004 \\
200,25 & 0.054 & 0.029 & 0.083 & 0.118 & 0.058 & 3.086 & 25.887 & 0.011 \\
200,50 & 0.090 & 0.104 & 0.194 & 0.207 & 0.187 & 4.734 & 37.423 & 0.003 \\
200,100 & 0.121 & 0.401 & 0.522 & 0.548 & 0.518 & 7.636 & 26.424 & 0.038 \\
500,3 & 0.827 & 0.004 & 0.831 & 0.849 & 0.813 & - & $>3600$ & 548.87 \\
500,10 & 0.973 & 0.029 & 1.002 & 1.016 & 0.992 & - & $>3600$ & 41.68 \\
500,25 & 1.076 & 0.150 & 1.226 & 1.248 & 1.214 & - & $>3600$ & 61.79 \\
500,50 & 0.818 & 0.610 & 1.428 & 1.459 & 1.401 & 220.83 & 997.65 & 3.82 \\
500,100 & 4.536 & 2.369 & 6.905 & 6.994 & 6.870 & 497.49 & 2294.8 & 0.165 \\
500,200 & 17.735 & 9.440 & 27.175 & 27.437 & 26.719 & 598.26 & 2122.8 & 26.44 \\
\hline
\end{tabular}

Table 7.4 - QAPV-VF2 comparison of processing time with almost-isomorphic pairs.

\begin{tabular}{|c|c|c|c|c|c|c|c|c|}
\hline \multirow{2}{*}{$\begin{array}{c}\text { Instance } \\
\text { n,d }\end{array}$} & \multicolumn{7}{|c|}{ QAPV } & \multicolumn{3}{c|}{ VF2 } \\
\cline { 2 - 9 } & avg dc* & avg qapv & $\Sigma$ avg & $\max$ & $\min$ & avg & $\max$ & $\min$ \\
\hline 100,3 & 0.010 & 0.000 & 0.010 & 0.021 & 0.006 & 0.454 & 2.372 & 0.000 \\
100,10 & 0.013 & 0.002 & 0.015 & 0.026 & 0.011 & 0.137 & 0.485 & 0.015 \\
100,25 & 0.016 & 0.007 & 0.023 & 0.032 & 0.021 & 0.442 & 1.596 & 0.010 \\
100,50 & 0.021 & 0.027 & 0.048 & 0.063 & 0.044 & 1.730 & 2.916 & 0.344 \\
200,3 & 0.038 & 0.001 & 0.039 & 0.049 & 0.034 & 68.863 & 368.28 & 0.446 \\
200,10 & 0.039 & 0.005 & 0.044 & 0.061 & 0.040 & 6.605 & 26.078 & 0.156 \\
200,25 & 0.053 & 0.003 & 0.056 & 0.084 & 0.033 & 4.409 & 18.404 & 0.434 \\
200,50 & 0.091 & 0.100 & $0 ; 191$ & 0.204 & 0.185 & 3.149 & 12.725 & 0.240 \\
200,100 & 0.120 & 0.392 & 0.512 & 0.530 & 0.506 & 18.533 & 39.091 & 3.281 \\
500,3 & 0.819 & 0.003 & 0.822 & 0.848 & 0.772 & - & $>3,600$ & 45,573 \\
500,10 & 0.975 & 0.026 & 1.001 & 1.018 & 0.991 & - & $>3,600$ & 10,617 \\
500,25 & 1.072 & 0.153 & 1.225 & 1.240 & 1.218 & - & $>3,600$ & 8,836 \\
500,50 & 0.822 & 0.603 & 1.426 & 1.453 & 1.405 & - & $>3,600$ & 20,519 \\
500,100 & 4.527 & 2.346 & 6.873 & 6.891 & 6.869 & 1215,46 & 1776,56 & 6,921 \\
500,200 & 17.787 & 9.370 & 27.157 & 27.215 & 27.029 & - & $>3,600$ & 117,87 \\
\hline
\end{tabular}

applying the function to a graph pair. Column 3 shows QAPV processing time of this pair and Column 4 shows the total average time for the pair (sum of Columns 2 and 3). Columns 5 (6) presents the sum of the corresponding maximum (minimum) values. These values do not necessarily correspond to the same pair; their sum indicates extremes for the processing time. 
The last three columns indicate the average, minimum and maximum processing times obtained by the algorithm VF2. Where an instance exceeded the time limit, the average time for its collection was not calculated.

\section{CONCLUSIONS AND DIRECTIONS FOR FUTURE WORK}

Within the orders and degrees studied, the results obtained are in accordance with Conjecture 4.1, the set of functions here proposed allowing us to discriminate between every graph pair used in the tests. We can see that $\boldsymbol{d} \boldsymbol{c}(\boldsymbol{G})$ had a complementary performance with respect to $\boldsymbol{w} \mathbf{3}(\boldsymbol{G})$ and $\boldsymbol{w} \mathbf{4}(G)$. On the other hand, $\boldsymbol{e s}(f(G))$ enhanced $\boldsymbol{w} \mathbf{3}(G)$ and $\boldsymbol{w} \mathbf{4}(G)$ performances in most cases at a very low additional cost.

We used regular graphs in the majority of the tests because they seem to present a more difficult problem than general graphs, owing to their structure restrictions. Nevertheless, general graphs could also be examined, the technique presenting no restriction concerning degree sequences, as it can be seen with the public instances we tested. For general graphs, es $(G)$ could be applied directly, which would mean much lower computing times.

The study shown here can be extended through the use of new weight function criteria and more efficient programming, especially for the walk-counting techniques. Interesting possibilities are brought by the matrices shown in [6]. It can be applied to other graph families, such as general planar graphs and trees. We also think that QAPV can be advantageously applied as a first resource to detect non-isomorphic pairs when generating given graph families, VF2 or another exact algorithm taking over the doubtful cases.

The relation between degree and number of centers for regular graphs (in $\boldsymbol{d c}(\boldsymbol{G})$ calculation) is a subject that could lead to interesting theoretical studies. However, they are not within the scope of this paper.

The es $(\boldsymbol{G})$ function, even when applied twice as in the planar examples, is very quick when compared with the QAP instance processing for the instances examined above 30,000 vertices.

The variance calculation times from Figure 4.2 are those of the original variance, the relaxed one being much less time-consuming. By starting with the relaxed instance, one can then expect to work with bigger graphs much more quickly.

Working with big planar graphs opens possibilities to apply the technique to pattern recognition problems, allowing for quantification of the differences between pairs of images.

It is interesting to observe that, if Conjecture 4.1 could be proven, this would be equivalent to establishing the complexity of the restricted graph isomorphism problem as being polynomial for all graphs.

The comparison with VF2 algorithm shows QAPV values as being much more rapid to calculate than VF2 results. Since VF2 is an exact constructive algorithm, this is not unexpected. On the other hand, QAPV can be applied to graph pairs of higher order than VF2. 


\section{REFERENCES}

[1] Abreu NMm, Boaventura-Netto PO, Querido TM \& GouvêA EF. 2002. Classes of quadratic assignment problem instances: isomorphism and difficulty measures using a statistical approach. Discrete Applied Mathematics, 124: 103-116.

[2] ARVIND V \& THORÁN J. 1985. Isomorphism testing: perspective and open problems. The Computational Complexity Column. Bulletin of the European Association for Theoretical Computer Science, 86: 66-84. In: http://theorie.informatik.uni-ulm.de/Personen/jt.html. Consulted June 2010.

[3] Angel R \& Zissimopoulos V. 1998. On the quality of the local search for the quadratic assignment problem. Discrete Applied Mathematics, 82: 15-25.

[4] Boaventura-Netto PO \& Abreu NMM. 1997. Cost solution average and variance for the quadratic assignment problem: a polynomial expression for the variance of solutions. Actas de Resumenes Extendidos, 408-413, I ELIO/Optima 97, Concepción, Chile.

[5] Bonventura-Netto PO. 2012. Grafos: Teoria, Modelos, Algoritmos (in Portuguese). $5^{\text {th }}$ ed., Edgard Blücher, São Paulo. http://www.po.coppe.ufrj.br/docentes/boaventura/home.html.

[6] Bessa AD, Rocha-Neto IC, Pinho StR, Andrade RFS \& Petit Lobão TC. 2012. Graph cospectrality using neighborhood matrices. Electronic Journal of Combinatorics, 19(3): 23.

[7] Cvetkovic D, Rowlinson P \& Simic S. 1997. Eigenspaces of graphs, Cambridge University Press, Cambridge.

[8] Cross ADJ, Wilson RC \& HANCOCK ER. 1997. Inexact graph matching using genetic search. Pattern Recognition, 30(6), 953-970.

[9] CZERWINSKI R. 2010. A polynomial time algorithm for graph isomorphism. http://arxiv.org/pdf/0711.2010v4. Consulted August 2011.

[10] DePiero F \& Krout D. 2003. An algorithm using length-r paths to approximate subgraph isomorphism. Pattern Recognition Letters, 24: 33-46.

[11] Ding H \& HuAng Z. 2009. Isomorphism identification of graphs: especially for the graphs of kinematic chains. Mechanism and Machine Theory, 44: 122-139.

[12] Douglas B. 2011. The Weisfeiler-Lehman method and graph isomorphism testing. http://arxiv.org/pdf/1101.5211v1. Consulted August 2011.

[13] Dharwadker A \& Tevet J. 2009. The graph isomorphism algorithm. In Proceedings of the Structure Semiotics Research Group. Eurouniversity Tallinn. Also in http://www.dharwadker.org/tevet/isomorphism/. Consulted June 2010.

[14] Foggia P, Sansone C \& Vento M. 2001. A performance comparison of five algorithms for graph isomorphism. Proc. of the $3^{\text {rd }}$. IAPR-TC-15 International Workshop on Graph-based Representations, Venice, Italy.

[15] Garey MR \& Johnson DS. 1979. Computers and intractability: a guide to NP-completedness. W.H. Freeman.

[16] Gori M, Maggini M \& SARTi L. 2001. Graph matching using random walks. Proc. of the $3^{\text {rd }}$. IAPR-TC-15 International Workshop on Graph-based Representations, Venice, Italy.

[17] Graves GW \& Whinston AB. 1970. An algorithm for the quadratic assignment problem. Management Science, 17: 453-471. 
[18] Gross JL \& Yellen J. (eds.) 2005. Handbook of graph theory, CRC Press LLC, Boca Raton.

[19] Harary F. 1971. Graph theory, Addison-Wesley, Reading, Massachussetts.

[20] Haemers WH \& SPENCE E. 2001. The pseudo-geometric graphs for generalized quadrangles of order .3. European J. Combin., 22(6): 839-845.

[21] IGRAPH LiBRARY. http://igraph.sourceforge.net/ Consulted June 2012.

[22] JAIN BJ \& WYSOTSKI F. 2005. Solving inexact graph isomorphism problems using neural networks. Neurocomputing, 63: 45-67.

[23] KnUth D. 1997. The art of computer programming, vol. 2, Seminumerical algorithms. $3^{\text {rd }}$ ed., Addison-Wesley, Reading, Massachussetts.

[24] Loiola EM, Abreu NMM, Boaventura-Netto PO, Hahn P \& Querido TM. 2007. A survey for the quadratic assignment problem. European J. of Operational Research, 176: 657-690.

[25] LeE L, RANGel MC \& Boeres MCS. 2007. Reformulação do problema de isomorfismo de grafos como o problema quadrático de alocação (in Portuguese), Anais do XXXIX SBPO, 1601-1612, Fortaleza.

[26] Melo VA, Boaventura-Netto PO, Hahn P \& Bahiense L. 2010. Graph isomorphism and QAP variances. Combinatorial optimization in practice, 8(2): 209-234.

[27] MCKaY B. 1981. Practical graph isomorphism. Congressus Numerantium, 30(1): 45-87.

[28] Marsaglia G. 1984. A current view of random number generators. Keynote address, Proc. $16^{\text {th }}$ Symposium on the Interface, Atlanta. Elsevier.

[29] Porumbel DC. 2011. Isomorphism testing via polynomial-time graph extensions. Journal of Mathematical Modelling and Algorithms, 10(2): 119-143.

[30] Presa JLL. 2009. Efficient algorithms for graph isomorphism testing. D.Sc. Thesis, Universidad Rey Juan Carlos, Madrid.

[31] Santos PLF. 2010. Teoria espectral de grafos aplicada ao problema de isomorfismo de grafos (in Portuguese). M.Sc. Dissertation, Department of Informatics, Federal University of Espírito Santo, Brazil.

[32] Santo M de, Foggia P, Sansone C \& Vento M. 2003. A large database of graphs and its use for benchmarking graph isomorphism algorithms. Pattern Recognition Letters, 24: 1067-1079.

[33] http://www.cs.sunysb.edu/ algorith/implement/vflib/implement.shtml. Consulted May 2012.

[34] Voss S \& SubhloK J. 2010. Performance of general graph isomorphism algorithms. Technical Report Number UH-CS-09-07, Department of Computer Science, University of Houston, Houston, TX, 77204, USA. http://www.cs.uh.edu/docs/cosc/technical-reports/2010/09_07.pdf. 